birds that performed poorly. Evidence for a connection between test performance and biological fitness could not have been assessed by the earlier approaches based on interspecies comparisons.

For any given individual, the bird's performance on each of the four skill tests was correlated. A principal-components statistical analysis, which identifies the number of factors accounting for variance in an array of scores, showed that a single unknown factor accounted for $65 \%$ of the variance in test scores. The authors refer to this factor as 'general intelligence', analogous to the ' $g$ factor' used to assess general intelligence in humans. However, it is worth noting that the magpie tests assess learning abilities rather than testing the capacity to invent creative solutions to problems - a talent sometimes considered to be a defining characteristic of animal or human intelligence.

Humphrey proposed that intelligence evolved in response to the pressures of social complexity. Ashton and colleagues, however, did not directly address this evolutionary hypothesis; instead, they investigated the relationship between social-group size and the development of cognition in the birds' early life, a linkage that was observed to emerge by the time the birds were 200 days old. Nevertheless, the link between cognitive prowess and fitness identified by Ashton and colleagues has major implications for connecting the social-intellect hypothesis to an underlying evolutionary mechanism, and it suggests that selection is acting on a relationship between sociality and cognition.

Group size is, of course, a crude index of social complexity, just as brain size is a crude indicator for the complexities of cognition being selected for. Indeed, group size itself cannot be the key causal factor - the immensity of a wildebeest herd, for example, is unlikely to select strongly for intellect. And even three individuals can suffice to create a high level of social complexity, as demonstrated in the humorous account provided by Jerome K. Jerome's 1889 book Three Men in a Boat, or documented in an analysis ${ }^{3}$ of three adult chimpanzees that repeatedly shifted alliances in a way that allowed each to be supreme for a while in their 'game of thrones'. Ashton and colleagues can only speculate on how magpies' cognitive powers relate to social-group size and number of offspring, and suggest that some kind of political skill, such as the ability to successfully handle or avoid conflicts, might be involved.

Which types of intelligence deserve closer attention in future studies of the socialintelligence hypothesis? Some substantially different ideas exist regarding which form of intelligence might be selected for ${ }^{4}$. One such contrast is between whether social complexity selects for overall intelligence across many different contexts (also known as domain-general intelligence), or for specialized forms of intelligence, such as the social skill of understanding what others might be thinking. This might sound like a tall order for a magpie. But there is evidence ${ }^{10}$ for such sophisticated behaviour in the crow family a western scrub-jay (Aphelocoma californica) might make a theft-prevention manoeuvre by relocating hidden food elsewhere if it spots that another bird observed where the food was hidden. Social cognition has numerous other manifestations ${ }^{11,12}$. Yet, what Ashton et al. ${ }^{2}$ tested was essentially non-social cognition. What now begs to be fleshed out is the nature of both the social and non-social intelligence skills that may have been at work in the phenomena these authors observed.

Andrew Whiten is in the Centre

for Social Learning and Cognitive

Evolution, School of Psychology and

Neuroscience, University of St Andrews,

St Andrews KY16 9JP, UK. e-mail:a.whiten@st-andrews.ac.uk

1. Humphrey, N. K. in Growing Points in Ethology (eds Bateson, P. P. G. \& Hinde, R. A.) 303-317 (Cambridge Univ. Press, 1976)

2. Ashton, B. J., Ridley, A. R., Edwards, E. K. \& Thornton, A. Nature 554, 364-367 (2018).

3. de Waal, F. B. M. Chimpanzee Politics: Power and Sex Among Apes (Cape, 1982).

4. Byrne, R. W. \& Whiten, A. Machiavellian Intelligence: Social Complexity and the Evolution of Intellect in Monkeys, Apes and Humans (Oxford Univ. Press, 1988).

5. Seyfarth, R. M. \& Cheney, D. L. Anim. Behav. 103, 191-202 (2015).

6. Dunbar, R. I. M. J. Hum. Evol. 28, 287-296 (1995)

7. Dunbar, R. I. M. \& Shultz, S. Science $\mathbf{3 1 7}$, 1344-1347 (2007).

8. Perez-Barberia, F. J., Shultz, S. \& Dunbar R. I. M. Evolution 61, 2811-2821 (2007).

9. Powell, L. E., Isler, K. \& Barton, R. A. Proc. R. Soc. B 284, 20171765 (2017).

10.Clayton, N. S., Dally, J. M. \& Emery, N. J. Phil. Trans. R. Soc. B 362, 507-522 (2007).

11.Emery, N. J., Clayton, N. S. \& Frith, C. D. Phil. Trans $R$. Soc. B 362, 485-488 (2007).

12. Whiten, A. \& van de Waal, E. Neurosci. Biobehav. Rev. 82, 58-75 (2017).

This article was published online on 7 February 2018.

\title{
NEUROSCIENCE
}

\section{Burst firing sets the stage for depression}

Salvos of neuronal activity in the brain's lateral habenula, regulated by astrocyte cells, drive depression-like behaviours in rodents. The finding might help us to understand one antidepressant and to develop more. SEE ARTICLES P.317 \& P.323

\section{WILLIAM M. HOWE \& PAUL J. KENNY}

$\mathrm{O}$ pposing forces shape our everyday lives - for instance, stimuli can encourage us to move or stop, and events can make us happy or sad. Accordingly, our brains are designed with 'yin-yang' systems that guide our actions and influence our feelings. Neurons in the brain's mesolimbic system promote reward-seeking behaviour and help to process information about actions that result in pleasurable outcomes ${ }^{1-3}$. By contrast, neurons in the lateral habenula ( $\mathrm{LHb}$ ) encode information related to noxious outcomes and suppress reward-seeking ${ }^{4-6}$. Unbalancing these opposing systems might therefore affect our behaviour. Indeed, emerging evidence ${ }^{7}$ suggests that LHb hyperactivity contributes to mood disorders such as major depression. Two papers ${ }^{8,9}$ in Nature now shed light on the mechanisms that underlie LHb hyperactivity, and on how the antidepressant drug ketamine modulates this state.

In the first paper, Yang and colleagues ${ }^{8}$ (page 317) assessed the firing activity of LHb neurons in two rat models of depression. Neuronal firing involves depolarization of the electrical potential across the cell membrane (in a resting state, the inside of the cell is negatively charged relative to the extracellular space around it). Hyperpolarization, in which the cell interior becomes more negative than normal, is typically associated with neuronal inhibition.

By studying brain slices ex vivo, Yang and coworkers showed that $\mathrm{LHb}$ neurons were more likely to fire in a pattern of rapid bursts in the 'depressed' rats than in control animals. They also observed that, when the LHb neurons were hyperpolarized, this increased the likelihood that these cells would fire in bursts rather than steady volleys. The researchers went on to show that they could increase depression-like behaviours in rats using a genetic manipulation to drive hyperpolarization, and so burst firing, in LHb neurons.

Next, the group investigated the signals that regulate this burst firing. In other brain regions ${ }^{10}$, burst firing is controlled by $\mathrm{N}$-methyl-D-aspartate receptors (NMDARs) - membrane-spanning channel proteins whose activation leads to an influx of positively charged calcium ions into neurons, resulting in depolarization and neuronal firing. Yang 
and colleagues found that burst firing of LHb neurons required the activity of NMDARs and of another class of protein, T-type voltagesensitive calcium channels (T-VSCCs).

Could inhibition of NMDARs prevent bursting? Ketamine is an NMDAR inhibitor and a promising, rapid-acting antidepressant in humans (taking effect in as little as $30 \mathrm{~min}$ utes) ${ }^{11}$ that is currently in clinical trials for the treatment of major depressive disorder with imminent risk of suicide. The mechanisms by which ketamine acts have been a puzzle to scientists. Strikingly, Yang et al. found that local infusion of ketamine into the LHb elicited antidepressant-like responses in depression-prone rats. These findings suggest that the therapeutic actions of ketamine might relate, at least in part, to its ability to block burst firing in the LHb.

In the second paper, Cui et al. ${ }^{9}$ (page 323) turned their attention to the mechanisms by which LHb neurons become skewed to firing in burst mode during depression. The authors performed a large-scale analysis of differentially expressed proteins in the LHb. This revealed that the expression of Kir4.1, a component of potassium-ion $\left(\mathrm{K}^{+}\right)$channels, was increased in the LHb of depression-prone rats compared with that of controls.

Kir4.1 is expressed in astrocytes, cells that interact with neurons to influence their activity state ${ }^{12}$ (although the functional relevance of such interactions is still being defined). The researchers showed that overexpression of Kir4.1 in LHb astrocytes increased the burst firing of local neurons and precipitated depression-like behaviours in mice. Conversely, reducing Kir4.1 expression in depression-prone rats reduced the burst firing of LHb neurons, and attenuated the animals' depression-like behaviours.

How do Kir4.1-containing channels in astrocytes regulate the activity of neurons? Neurons can pump $\mathrm{K}^{+}$from their cytoplasm into the extracellular space to cause hyperpolarization. Cui et al. provide evidence that astrocytic $\mathrm{K}^{+}$channels in the LHb help to clear away extracellular $\mathrm{K}^{+}$. This facilitates the ability of LHb neurons to enter a hyperpolarized state, and hence to fire in bursts (Fig. 1). Future studies will be required to understand whether $\mathrm{LHb}$ astrocytes interact with neurons in other ways to influence their patterns of activity.

Together, these two papers provide crucial insights into a depression-associated pattern of cell firing in the $\mathrm{LHb}$ and its regulation by ketamine. However, exactly why burst firing of LHb neurons increases depression-like behaviours in rodents remains unclear. One potential explanation, put forward by Yang et al., is the inhibitory influence that LHb neurons exert over reward-associated dopamine neurons in the mesolimbic system and mood-associated serotonin neurons in the midbrain. Perhaps burst firing of $\mathrm{LHb}$ neurons alters the activity of these downstream neurotransmitter systems in a manner that reduces their positive effects
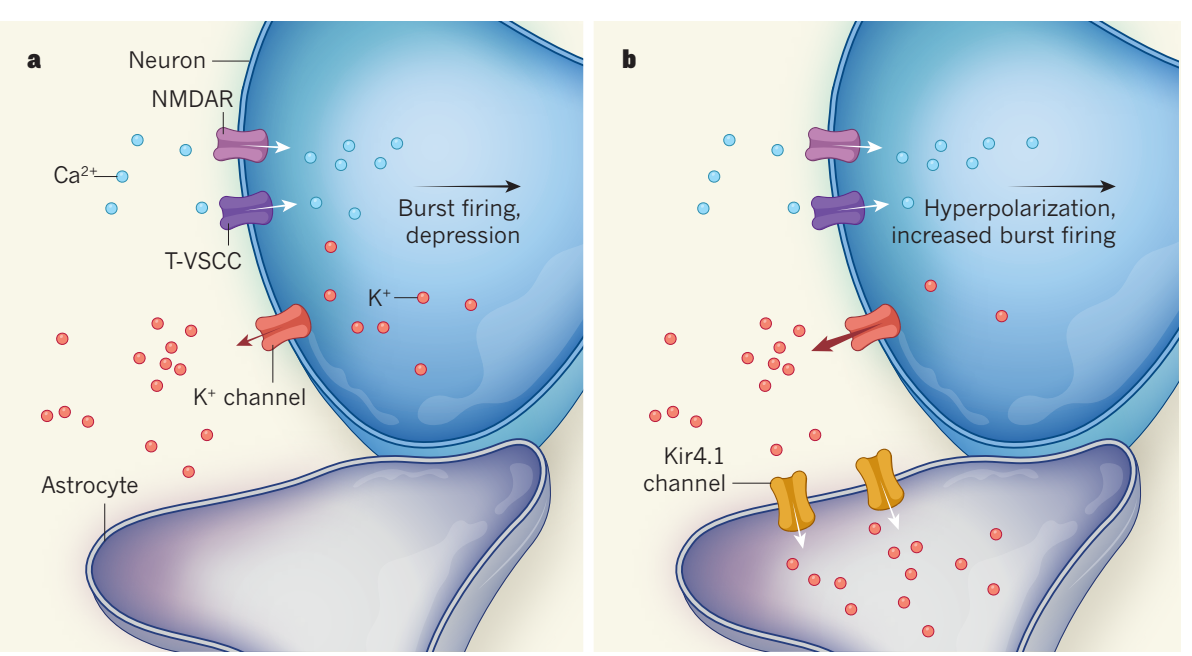

Figure 1 | Burst firing in depression. a, The activation of membrane-spanning $N$-methyl-D-aspartate receptors (NMDARs) leads to an influx of calcium ions $\left(\mathrm{Ca}^{2+}\right)$ that depolarizes neurons (the insides of which are negative relative to the outside in resting conditions), and causes firing. $\mathrm{Ca}^{2+}$ influx also leads to an efflux of potassium ions $\left(\mathrm{K}^{+}\right)$through $\mathrm{K}^{+}$-channel proteins. Yang et al. ${ }^{8}$ show that, in the brain's lateral habenula (LHb), coordinated activity of NMDARs and T-type voltage-sensitive calcium channels (T-VSCCs) causes neuron firing to occur in a pattern of rapid bursts, and that such firing leads to depression-like symptoms in rats through unknown mechanisms. b, Cui et al. ${ }^{9}$ demonstrate that $\mathrm{K}^{+}$ions are rapidly cleared away by nearby cells called astrocytes, which express $\mathrm{K}^{+}$channels containing the protein Kir4.1. This facilitates rapid $\mathrm{K}^{+}$efflux from the neuron and its entry into a state of hyperpolarization, which increases the likelihood of burst firing.

on mood, thereby increasing vulnerability to depression. Testing this possibility, and other potential mechanisms, will require further experimentation. The potential involvement of subpopulations of dopamine and serotonin neurons that encode aversion-relevant stimuli must also be considered ${ }^{13}$.

The current studies have several therapeutic implications. First, Cui and colleagues' data show that astrocytes might have a key role in regulating a brain system involved in mood and motivation. Previous studies linking astrocyte function to disease states have focused mostly on neurodegenerative and developmental disorders ${ }^{14,15}$, but the current papers suggest that modulating the activity of these cells might be a way to treat psychiatric disorders.

Second, Yang et al. showed that a T-VSCC blocker delivered directly into the LHb had antidepressant-like effects, similar to those of ketamine. This raises the exciting possibility that T-VSCC blockers, or other compounds that suppress LHb burst firing, could be effective antidepressants.

Finally, the papers shed light on the possible mechanisms by which ketamine elicits rapid antidepressant effects in humans. Ketamine and its metabolites stimulate the formation of synaptic connections between neurons in the brain ${ }^{16-18}$, a process that is thought to be important for the drug's therapeutic effects. The findings suggest that another property of the drug - its ability to inhibit burst firing in a brain region implicated in aversion and negative mood - also contributes to its efficacy and explains its rapid onset of action. This knowledge might facilitate the development of next-generation ketamine-related antidepressants that specifically target LHb activity and that might eliminate two major side effects of ketamine and other NMDAR blockers: their abuse potential and the induction of a transient, schizophrenia-like, psychotic state.

William M. Howe and Paul J. Kenny are in the Department of Neuroscience, Icahn School of Medicine at Mount Sinai, New York, New York 10029, USA.

e-mail:paul.kenny@mssm.edu

1. Berridge, K. C. \& Robinson, T. E. Brain Res. Brain Res. Rev. 28, 309-369 (1998).

2. Di Chiara, G. \& Bassareo, V. Curr. Opin. Pharmacol. 7, 69-76 (2007).

3. Schultz, W. Neuron 36, 241-263 (2002).

4. Christoph, G. R., Leonzio, R. J. \& Wilcox, K. S. J. Neurosci. 6, 613-619 (1986).

5. Ji, H. \& Shepard, P. D. J. Neurosci. 27, 6923-6930 (2007).

6. Matsumoto, M. \& Hikosaka, O. Nature $\mathbf{4 4 7}$, 1111-1115 (2007)

7. Sartorius, A. et al. Biol. Psychiatry 67, e9-e11 (2010).

8. Yang, Y. et al. Nature 554, 317-322 (2018).

9. Cui, Y. et al. Nature 554, 323-327 (2018).

10.Grace, A. A., Floresco, S. B., Goto, Y. \& Lodge, D. J. Trends Neurosci. 30, 220-227 (2007).

11.Berman, R. M. et al. Biol. Psychiatry 47, 351-354 (2000).

12. Halassa, M. M. \& Haydon, P. G. Annu. Rev. Physiol. 72, 335-355 (2010)

13.Lammel, S., Ion, D. I., Roeper, J. \& Melenka, R. C. Neuron 70, 855-862 (2011).

14. Molofsky, A. V. et al. Genes Dev. 26, 891-907 (2012).

15. Ransom, B., Behar, T. \& Nedergaard, M. Trends Neurosci. 26, 520-522 (2003).

16.Autry, A. E. et al. Nature 475, 91-95 (2011).

17.Zanos, P. et al. Nature 533, 481-486 (2016).

18. Li, N. et al. Science 329, 959-964 (2010). 